\title{
Genetic analyses of anthocyanin content using polyploid GWAS followed by QTL detection in the sweetpotato (Ipomoea batatas L.) storage root
}

\author{
Emdadul Haque ${ }^{1}$, Eiji Yamamoto ${ }^{2}$, Kenta Shirasawa ${ }^{2}$, Hiroaki Tabuchi ${ }^{1}$, Ung-Han Yoon ${ }^{3}$, \\ Sachiko Isobe ${ }^{2}$ and Masaru Tanaka ${ }^{1}$ \\ ${ }^{1}$ Kyushu Okinawa Agricultural Research Center, NARO, 6651-2 Yokoichi-cho, Miyakonojo, Miyazaki 885-0091, \\ Japan \\ ${ }^{2}$ Kazusa DNA Research Institute, 2-6-7 Kazusakamatari, Kisarazu, Chiba 292-0818, Japan \\ ${ }^{3}$ National Institute of Agricultural Sciences, 370 Nongsaengmyeong-ro, Jeonju 54874, Korea
}

Corresponding author: M. Tanaka, E-mail: mtanaka@affrc.go.jp, Phone: +81-986-24-4274, Fax: +81-986-24-4283

Received on March 26, 2020; Accepted on July 30, 2020

\begin{abstract}
Genetic studies on the purple-fleshed sweetpotato (Ipomoea batatas L.), which is rich in anthocyanin (AN) in the storage root, were performed by polyploid GWAS based on the allele dosage probability using 59,675 SNPs obtained from $94 \mathrm{~F}_{1}$ progenies between the cultivars 'Konaishin' (which has a high yield but no AN) and 'Akemurasaki' (which has a high AN content but low to moderate yield). The distribution of relative AN content was highly biased, with $60 \%$ of clones showing a low to undetectable level $\left(A_{530}<0.5\right)$. Fifty-nine SNPs from six signals on homologous groups (HGs) 3, 5 (one major and one smaller signal), 7,13 , and 15 were strongly associated with the relative AN content. Twelve SNPs from the major signal and one from the smaller signal of HG 5 were further detected by QTL analysis. In a database search of the AN biosynthesis gene, transcription factors $I b M Y B 1$ and $I b W D 40$ and AN structural genes $I b F 3 H$ and $I b D F R$ were located on HG 5 , suggesting that an SNP marker or markers from HG 5 might be tightly linked to candidate gene(s) homologous to one of these transcription factors and $A N$ structural genes as a major factor in determining AN accumulation in the storage roots. These results would enhance our understanding of the underlying genetic basis of $\mathrm{AN}$ accumulation in the storage roots of sweetpotatoes, and the SNP markers found here, especially 13 SNPs from HG 5, would be a potential platform for future marker-assisted selection for breeding high-AN sweetpotato varieties.
\end{abstract}

Keywords: anthocyanin, ddRAD SNPs, polyploid GWAS, QTLs, root drymatter, sweetpotato

\section{Introduction}

The sweetpotato (Ipomoea batatas (L.) Lam), the world's seventh most important food crop (FAO 2018), is increasingly used in the food industry. Anthocyanin (AN), a color pigment which belongs to a class of flavonoid synthesized via the phenylpropanoid pathway, is a major functional component of sweetpotato storage roots. Sweetpotato storage roots with purple flesh contain a majority of ANs, whereas those with other flesh colors such as white, yellow, or orange contain little or no AN (Tanaka et al. 2017). In Japan, purple-fleshed sweetpotatoes rich in AN have been used for various purposes, for example as principal sources of natural food colorant due to their superior color characteristics, for table use, in 'shochu' (sweetpotato liquor) production, and for their high value added to a wide range of food items because of their potential ability to counteract some lifestyle-related diseases (Tanaka et al. 2017). In Japan, improvements of the AN-rich purple-fleshed sweetpotato have been a key breeding interest, and several cultivars have already been developed (Sakai et al. 2010, Katayama et al. 2017, Tanaka et a1. 2017). Because of the heterozygosity and hexasomic hexaploid nature of the sweetpotato, leading to diverse combinations of parental chromosomes in the male and female

Haque E, Yamamoto E, Shirasawa K, Tabuchi H, Yoon U-H, Isobe S, Tanaka M 2020 Genetic analyses of anthocyanin content using polyploid GWAS followed by QTL detection in the sweetpotato (Ipomoea batatas L.) storage root. Plant Root 14:11-21. doi:10.3117/plantroot.14.11

Copyrights 2020, Plant Root (JSRR), www.plantroot.org 
gametes, in these breeding programs, one cultivar is released every 10 years on average through extensive field evaluation of a large number of seedlings in the early stage of breeding and consecutive line selection (Kumagai 2001, Sakai et al. 2010).

Molecular markers, which are diagnostic of alleles of genes linked to favorable traits, are expected to dramatically reduce the labor and time required to screen clones in the early stage of the conventional breeding program, thus shortening the breeding period and finding promising clones from wider genetic variations. These molecular markers can be identified through molecular genomics and genetic study which has been employed for several molecular breeding strategies, including highdensity genetic mapping enabling quantitative trait locus (QTL) identification or association mapping (e.g. GWAS). While the technology is efficient in diploid species, the hexaploidy genome and its allogamous nature $(2 n=6 x=90)$ have become the main obstacles for genetic analysis and DNA marker development in the sweetpotato. Although efforts were made with relatively few AFLP (CervantesFlores et al. 2011, Nakayama et al. 2012), with AFLP and SSR (Zhao et al. 2013, Yu et al. 2014), with SSR (Zhang et al. 2016, Yada et al. 2017), and in a recent study, with SNP (Gemenet et al. 2020) DNA marker-based genetic studies on agronomic traits of the sweetpotato, to date no genetic studies on the development of DNA markers that enable efficient selection in AN-rich purple-fleshed sweetpotato breeding, i.e., marker-assisted selection, have been reported.

In hexasomic hexaploid sweetpotatoes, a firsthand high-density SNP genetic map (with 28,087 double-simplex SNPs, a total distance of $33,020.4 \mathrm{cM}$, and a marker density of $1.17 \mathrm{cM}$ ) based on NGS technology was reported by Shirasawa et al. (2017) after developing a draft genome sequence of diploid I. trifida (Hirakawa et al. 2015). In one example of genetic research on the sweetpotato, we have successfully studied highdensity linkage map-based QTL analysis followed by GWAS to identify the responsive SNPs of several sweetpotato traits such as nematode resistance (Sasai et al. 2019), $\beta$-carotene, dry-matter content and starch content (Haque et al. 2020). However, these studies were limited to the mode of inheritance of Mendelian markers resembling that of the genetic markers in the diploid species I. trifida (Shirasawa et al. 2017, Sasai et al. 2019). Updated methods in NGS analysis enabling integrated pseudochromosomes and genetic mapping enabling the estimation of multiple-dosage markers in hexasomic hexaploidy sweetpotatoes are a prerequisite for GWAS or QTL analysis.
In the current study, we have taken advantage of new bioinformatic tools that were developed for polyploid genetics in the form of 'NGS-based SNP analysis' to generate a completely integrated SNP dataset in 15 pseudo-homologous groups (HGs) for both parents instead of an independent HG for each parent (TRAS sweetpotato genome sequencing consortium 2019). We have also made use of 'polyploid GWAS' using the allele dosage probability of each SNP calculated from the read count of NGS data, thus enabling the detection of the allele-dose-dependent relation between SNPs and target traits, which could not be detected using the previous GWAS method involving only homo and hetero genotypes of each SNP (Yamamoto et al. 2020). Thus, the initial goal of this study is to utilize polyploid GWAS to identify a wider range of SNP loci linked to storage root AN with the aim of enhancing our understanding of the underlying genetic basis of AN accumulation in the storage roots of sweetpotatoes. Next, we provide a useful resource base for the development of molecular markers from these candidate SNP loci, with the prime goal of a marker-assisted selection program. We quantified the relative $\mathrm{AN}$ content in a segregating population consisting of $94 \mathrm{~F}_{1}$ progenies between the cultivars 'Konaishin' and 'Akemurasaki'. We report on the polyploid GWAS method using 59,675 SNPs and compare the GWAS result with QTL analysis.

\section{Materials and Methods}

\section{Plant materials and growing condition}

I. batatas cv. Konaishin (KNS) and cv. Akemurasaki $(\mathrm{AKM})$ and $94 \mathrm{~F}_{1}$ progenies (hereafter referred to as $\mathrm{KAF}_{1}$ ) were used for this study. KNS is a novel cultivar for starch production released in 2018 with no AN content and extraordinarily high yield. On the other hand, AKM is a cultivar with significantly high AN content (Sakai et al. 2010), low to moderate yield, and moderate starch content.

Seedling multiplication, pot cultivation and harvesting of roots of KNS, AKM and their $\mathrm{KAF}_{1}$ were carried out under greenhouse conditions in Kyushu Okinawa Agricultural Research Center, NARO (KARC/NARO), Miyakonojo, Miyazaki prefecture, Japan, in 2018 as described previously (Haque et al. 2020). In brief, for seedling multiplication, stem cuttings of the lateral branches were nurtured in a temperaturecontrolled greenhouse. For developing storage roots, pot cultivation was carried out in another greenhouse using 6L plastic pots (Kaneya Co. LTD., Aichi, Japan, height $20.5 \mathrm{~cm} \times$ inner 
diameter $20.5 \mathrm{~cm}$ ) from July to November in 2018. The experiment was divided into three-unit plots, each containing 96 pots. Randomization was done within the plots. Watering was done every day using a shower for the first seven days and followed by $500 \mathrm{~mL}$ of water per pot twice a week. Rotation of the pots was conducted weekly to minimize the differences in the microenvironment. Air temperature and soil temperature $(7 \mathrm{~cm}$ below the soil surface) were recorded on a monthly basis from three spots in the green house with a thermo recorder (T\&D Co. LTD., Matsumoto, Japan). There was no temperature difference among the three locations in the greenhouse. The average monthly minimum/maximum daily temperatures (data from the center spot in the greenhouse) of the air and soil were $23.7 / 45.5$ and $25.5 / 42.1,20.7 / 45.8$ and $22.9 / 41.0,21.6 / 34.7$ and $23.3 / 31.3,13.2 / 29.2$ and $15.2 / 28.9$, and $9.1 / 25.9$ and $11.3 / 25.0^{\circ} \mathrm{C}$ for July, August, September, October, and November, respectively.

\section{Measurement of relative AN content}

Two to four medium-sized storage roots were selected for the measurement of relative AN content. Then the thickest parts of the selected storage roots (approximately $40 \mathrm{~g} /$ plant) with skin (the relative AN content of the skin was very low) were cut into fine strips with a food processor (slicer coupled thinner) (Fig. 1 shows the storage root flesh color in handmade slices). First, AN was extracted from $2.0 \mathrm{~g}$ of the sliced sample in $20 \mathrm{~mL}$ of $0.5 \%$ sulfuric acid contained in a $50 \mathrm{~mL}$ Falcon tube. Samples were kept at $4^{\circ} \mathrm{C}$ in a dark chamber. For the measurement of relative $\mathrm{AN}, 0.5 \mathrm{~mL}$ of sample was diluted (8 times) into MCILVAINE buffer $(0.1 \mathrm{M}$ citric acid, $0.2 \mathrm{M} \mathrm{Na}_{2} \mathrm{HPO}_{4}, \mathrm{pH}$ 3.0). After gentle mixing with vortexing, the relative $\mathrm{AN}$ content was analyzed as absorbance at $530 \mathrm{~nm}\left(\mathrm{~A}_{530}\right)$ using a Pharma spectrophotometer (UV-1700, Shimadzu Co., Kyoto, Japan).

ddRAD-Seq analysis to generate SNP loci in 15 pseudochromosomes

ddRAD-Seq analysis was performed according to the method of Shirasawa et al. (2016). In sequence analysis, a total of $471,617,154$ bases were obtained. The average number of bases per individual was $4,912,679$ (1.0\%). The mapping rate to the I. trifida pseudomolecule (TRAS sweetpotato genome sequencing consortium 2019) was $84.8 \%$ on average. Based on the sequence alignment data, 104,881 candidate SNP loci were identified after filtering. These SNPs were further filtered with maxmissing (the percentage of individuals that were called out of all individuals). A total of 59,675 SNPs derived using a max-missing value of $0.8(20 \%)$ were selected for further analyses.

The HiSeq reads of ddRAD-Seq libraries are

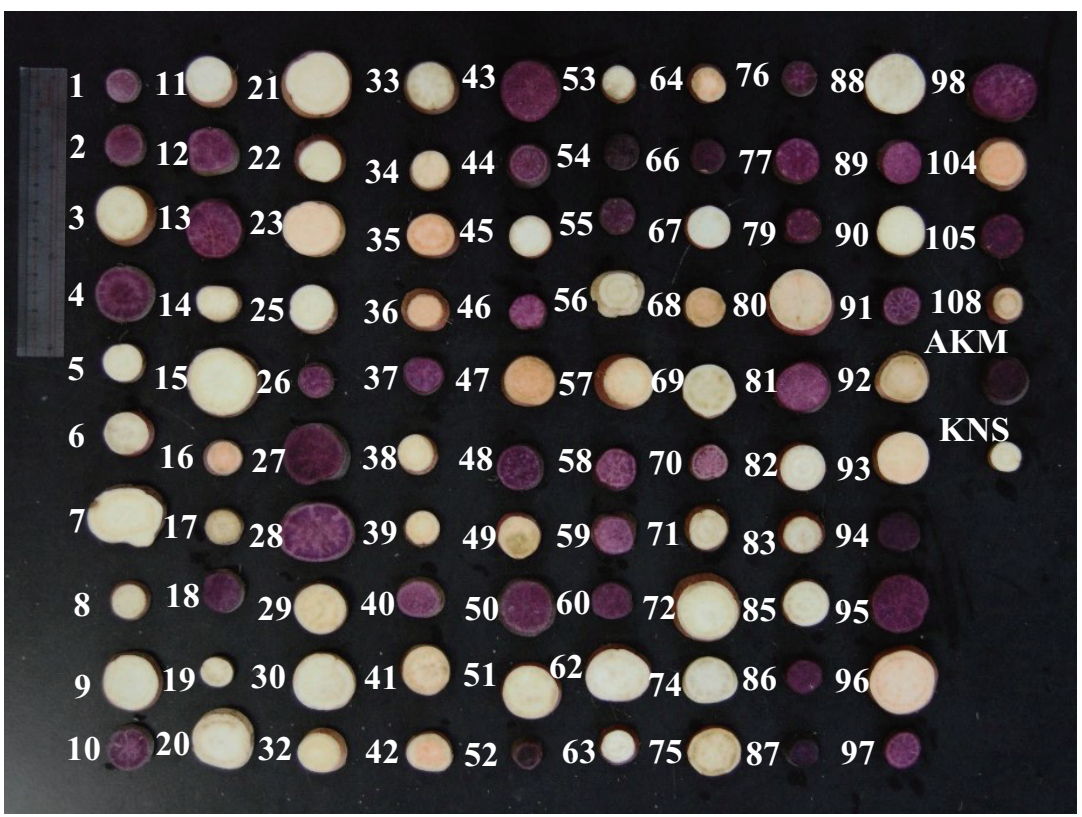

Fig. 1. Images of sliced storage roots in the Konaishin $(\mathrm{KNS}) \times$ Akemurasaki $(\mathrm{AKM})$ progenies $\left(\mathrm{KAF}_{1}\right)$. Photographs are from one replication as a representative of three replications. 
available in the DDBJ Sequence Read Archive under accession number DRA009153.

\section{Polyploid GWAS}

A total of 59,675 SNPs underwent allele dosage estimation using the method described previously (Yamamoto et al. 2020) with the following criteria: ploidy $=6$, minimum read depth $(\mathrm{dp})=10$, maximum $\mathrm{dp}=1000$, maximum missing $=0.5$, maximum frequency $=0.95$, round up $=1.00$, cut off $=0.05$, and read error probability $=0.001$. The resulting 59,649 SNPs were then subjected to GWAS according to the method described (Yamamoto et al. 2020). The Manhattan and quantile-quantile (QQ) plots were created with 'manhattan' and 'qq', respectively, in qqman of R (Turner 2014). The $P$-values of the SNPs from GWAS underwent sequential Bonferroni correction, where an adjusted $P$-value of 0.05 was used to set the significant threshold level.

\section{QTLs mapping}

SNP sites with $\mathrm{dp}>10$ and an altered allele frequency $(\mathrm{AAF}) \geq 0.7500$ and $\leq 0.2500$ were selected as simplex and double-simplex candidates. These SNPs were subjected to a chi-square test for the expected ratio of $1: 1$ or $3: 1$. A total of $28,492(47.7 \%)$ simplex and double-simplex loci fit into the above two segregation patterns. A genetic map for pseudochromosomes from both parents was constructed with Lep-MAP3 (v.3.0) (logarithm of odds $($ LOD) > 3.685) (Rastas 2017). The workflow of LepMAP3 consists of the modules ParentCall2, Filtering2, SeparateChromosomes2, JoinSingles2All, and OrderMarkers2. In the Filtering2 command, markers were filtered based on, e.g., high segregation distortion (data tolerance $=1$ ) and the amount of missing data.
The parameters used for the SeparateChromosomes2 command were: lodLimit $=3.685$ distortionLod $=1$ informativeMask $=123$ sizeLimit $=10$ theta $=0.05$.

QTLs analyses were done according to Haque et al. (2020) using MapQTL6.0 software. A LOD of 2.5 was selected as a threshold to determine the QTL.

Searching the location of structural genes (SGs) and their transcription factors (TFs) of the AN biosynthesis pathway within the reference genome

To identify the homology regions of SGs associated with the AN biosynthesis pathway, the $\mathrm{IbCHS}$ cDNA (AB023791), IbCHI cDNA (EU402467), $I b F 3$ ' $H$ cDNA (HM460342), IbF3H cDNA (HQ441168), IbDFR cDNA (EF108570), IbANS cDNA (GU598212, FJ478179), and IbUF3GT cDNA (KF056328) were used to query the reference genome database of I. trifida (ITR_r2.2. scaffold) using a local blast search (Genetyx ver.15, GENETYX Co.). For TFs associated with the AN biosynthesis pathway, the IbMYBI cDNA (AB258984), IbWD40 cDNA (JQ955738), and IbbHLH3 cDNA (KU589265) were used as query sequences. The highly homologous regions for each $\mathrm{SG}$ or TF from the reference genome were then confirmed using a BLAST search against the GenBank nucleotide database.

\section{Results}

\section{Distribution of AN content in the storage roots}

The distribution of relative AN content was highly biased, with $60 \%(n=56)$ of clones showing a low to undetectable level $\left(\mathrm{A}_{530}<0.5\right)$ (Fig. 2). The average $A_{530}$ values were $12.01 \pm 0.70$ and $0.10 \pm 0.01$ for AKM and KNS, respectively. The segregation

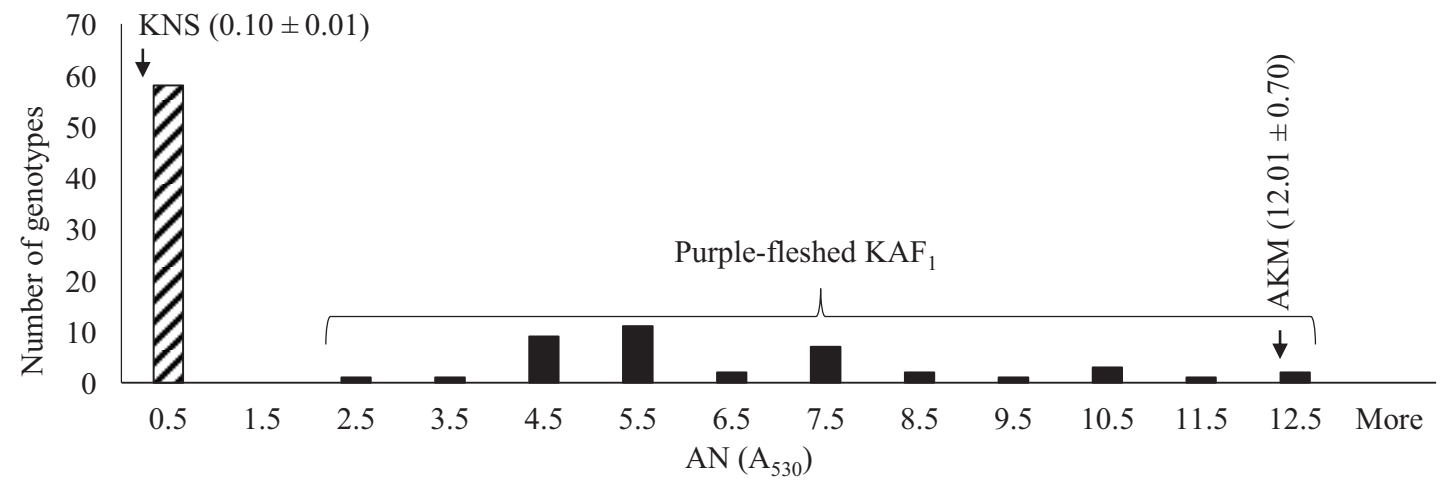

Fig. 2. Frequency distribution of the relative anthocyanin content $\left(A N ; A_{530}\right)$ in the $A K M \times K N S$ progenies $(n=94)$ and both parents. Arrows show the values (mean $\pm \mathrm{SD})$ of AKM $(12.01 \pm 0.70)$ and KNS $(0.10 \pm 0.01)$. 
Table 1. Summary of the 59 SNPs above the qqman significance thresholds

\begin{tabular}{|c|c|c|c|c|c|}
\hline Marker $^{\mathrm{a}}$ & $\mathrm{HGs}^{\mathrm{b}}$ & $-\log _{10}(P)$ & $P$-value & $\begin{array}{c}\text { Estimated allele } \\
\text { dosage }^{c}\end{array}$ & $\begin{array}{l}\text { Lep-MAP mapping } \\
\text { result }^{\mathrm{d}}\end{array}$ \\
\hline Itr_chr05_27090704 & 5 & 21.95 & $1.11 \times 10^{-22}$ & $\mathrm{~m}$ & \\
\hline Itr_chr05_26718188 & 5 & 21.55 & $2.84 \times 10^{-22}$ & $\mathrm{~m}$ & \\
\hline Itr_chr05_26718670 & 5 & 19.59 & $2.55 \times 10^{-20}$ & $\mathrm{~m}$ & \\
\hline Itr_chr05_26396741 & 5 & 17.99 & $1.03 \times 10^{-18}$ & $\mathrm{~s}$ & Mapped \\
\hline Itr_chr05_26029224 & 5 & 17.41 & $3.89 \times 10^{-18}$ & s & Mapped \\
\hline Itr_chr05_25701591 & 5 & 15.77 & $1.69 \times 10^{-16}$ & $\mathrm{~s}$ & Mapped \\
\hline Itr_chr05_24862401 & 5 & 14.76 & $1.75 \times 10^{-15}$ & $\mathrm{~m}$ & \\
\hline Itr_chr05_27879726 & 5 & 14.65 & $2.22 \times 10^{-15}$ & $\mathrm{~m}$ & \\
\hline Itr_chr05_26275802 & 5 & 13.83 & $1.47 \times 10^{-14}$ & $\mathrm{~m}$ & \\
\hline Itr_chr05_26275799 & 5 & 13.83 & $1.50 \times 10^{-14}$ & $\mathrm{~m}$ & \\
\hline Itr_chr05_26275800 & 5 & 13.66 & $2.17 \times 10^{-14}$ & $\mathrm{~m}$ & \\
\hline Itr_chr05_26275798 & 5 & 13.63 & $2.36 \times 10^{-14}$ & $\mathrm{~m}$ & \\
\hline Itr_chr05_26701979 & 5 & 13.33 & $4.68 \times 10^{-14}$ & $\mathrm{~m}$ & \\
\hline Itr_chr05_26275794 & 5 & 13.20 & $6.29 \times 10^{-14}$ & $\mathrm{~m}$ & \\
\hline Itr_chr05_23536069 & 5 & 13.15 & $7.06 \times 10^{-14}$ & $\mathrm{~s}$ & Mapped \\
\hline Itr_chr05_26701812 & 5 & 13.13 & $7.44 \times 10^{-14}$ & $\mathrm{~m}$ & \\
\hline Itr_chr05_26351002 & 5 & 12.84 & $1.43 \times 10^{-13}$ & $\mathrm{~s}$ & Not mapped \\
\hline Itr_chr05_27427060 & 5 & 12.84 & $1.44 \times 10^{-13}$ & s & Not mapped \\
\hline Itr_chr05_27569615 & 5 & 12.65 & $2.25 \times 10^{-13}$ & $\mathrm{~m}$ & \\
\hline Itr_chr05_22832191 & 5 & 12.59 & $2.57 \times 10^{-13}$ & $\mathrm{~s}$ & Mapped \\
\hline Itr_chr05_23551173 & 5 & 12.55 & $2.85 \times 10^{-13}$ & $\mathrm{~s}$ & Mapped \\
\hline Itr_chr05_29358196 & 5 & 11.90 & $1.26 \times 10^{-12}$ & $\mathrm{~S}$ & Mapped \\
\hline Itr_chr05_24960141 & 5 & 11.89 & $1.30 \times 10^{-12}$ & $\mathrm{~m}$ & \\
\hline Itr_chr05_26864099 & 5 & 11.45 & $3.56 \times 10^{-12}$ & $\mathrm{~m}$ & \\
\hline Itr_chr05_26550117 & 5 & 11.30 & $5.04 \times 10^{-12}$ & $\mathrm{~m}$ & \\
\hline Itr_chr05_29385059 & 5 & 11.00 & $9.90 \times 10^{-12}$ & $\mathrm{~m}$ & \\
\hline Itr_chr05_26550111 & 5 & 10.85 & $1.41 \times 10^{-11}$ & $\mathrm{~m}$ & \\
\hline Itr_chr05_29394223 & 5 & 10.75 & $1.77 \times 10^{-11}$ & $\mathrm{~s}$ & Mapped \\
\hline Itr_chr05_24861975 & 5 & 10.17 & $6.70 \times 10^{-11}$ & ds & Mapped \\
\hline Itr_chr05_26461536 & 5 & 10.16 & $6.96 \times 10^{-11}$ & $\mathrm{~s}$ & Mapped \\
\hline Itr_chr13_13144677 & 13 & 9.86 & $1.38 \times 10^{-10}$ & s & Not mapped \\
\hline Itr_chr13_13144678 & 13 & 9.74 & $1.83 \times 10^{-10}$ & $\mathrm{~S}$ & Not mapped \\
\hline Itr_chr05_23460474 & 5 & 9.64 & $2.30 \times 10^{-10}$ & $\mathrm{~m}$ & \\
\hline Itr_chr05_25731262 & 5 & 9.63 & $2.32 \times 10^{-10}$ & $\mathrm{~m}$ & \\
\hline Itr_chr13_13144896 & 13 & 9.32 & $4.79 \times 10^{-10}$ & $\mathrm{~S}$ & Not mapped \\
\hline Itr_chr05_29451144 & 5 & 9.26 & $5.49 \times 10^{-10}$ & $\mathrm{~m}$ & \\
\hline Itr_chr05_27696681 & 5 & 9.14 & $7.19 \times 10^{-10}$ & $\mathrm{~s}$ & Not mapped \\
\hline Itr_chr05_24605925 & 5 & 9.01 & $9.74 \times 10^{-10}$ & $\mathrm{~m}$ & \\
\hline Itr_chr05_24750065 & 5 & 9.00 & $9.98 \times 10^{-10}$ & $\mathrm{~m}$ & \\
\hline Itr_chr05_24927289 & 5 & 8.82 & $1.52 \times 10^{-09}$ & $\mathrm{~s}$ & Mapped \\
\hline Itr_chr05_24927560 & 5 & 8.73 & $1.85 \times 10^{-09}$ & s & Mapped \\
\hline
\end{tabular}




\begin{tabular}{|c|c|c|c|c|c|}
\hline Itr_chr05_25375649 & 5 & 8.63 & $2.32 \times 10^{-09}$ & $\mathrm{~m}$ & \\
\hline Itr_chr05_25375645 & 5 & 8.58 & $2.63 \times 10^{-09}$ & $\mathrm{~m}$ & \\
\hline Itr_chr05_28447653 & 5 & 8.39 & $4.08 \times 10^{-09}$ & ds & Not mapped \\
\hline Itr_chr05_26550141 & 5 & 8.15 & $7.10 \times 10^{-09}$ & ds & Not mapped \\
\hline Itr_chr05_25439632 & 5 & 8.09 & $8.07 \times 10^{-09}$ & $\mathrm{~m}$ & \\
\hline Itr_chr05_23484740 & 5 & 7.50 & $3.17 \times 10^{-08}$ & $\mathrm{~s}$ & Not mapped \\
\hline Itr_chr15_8725114 & 15 & 6.89 & $1.30 \times 10^{-07}$ & ds & Not mapped \\
\hline Itr_chr03_30274266 & 3 & 6.56 & $2.78 \times 10^{-07}$ & $\mathrm{~s}$ & Not mapped \\
\hline Itr_chr05_27388420 & 5 & 6.45 & $3.56 \times 10^{-07}$ & $\mathrm{~m}$ & \\
\hline Itr_chr05_27388642 & 5 & 6.32 & $4.73 \times 10^{-07}$ & $\mathrm{~m}$ & \\
\hline Itr_chr05_23460534 & 5 & 6.30 & $4.97 \times 10^{-07}$ & $\mathrm{~m}$ & \\
\hline Itr_chr05_27835691 & 5 & 6.28 & $5.22 \times 10^{-07}$ & ds & Not mapped \\
\hline Itr_chr05_27388444 & 5 & 6.23 & $5.89 \times 10^{-07}$ & $\mathrm{~m}$ & \\
\hline Itr_chr05_28124449 & 5 & 6.12 & $7.59 \times 10^{-07}$ & ds & Not mapped \\
\hline Itr_chr05_28508098 & 5 & 6.07 & $8.49 \times 10^{-07}$ & ds & Not mapped \\
\hline Itr_chr05_27388441 & 5 & 6.05 & $8.83 \times 10^{-07}$ & $\mathrm{~m}$ & \\
\hline Itr_chr07_17548345 & 7 & 6.03 & $9.29 \times 10^{-07}$ & $\mathrm{~s}$ & Not mapped \\
\hline Itr_chr05_7761458 & 5 & 6.01 & $9.73 \times 10^{-07}$ & ds & Mapped \\
\hline \multicolumn{6}{|c|}{$\begin{array}{l}\text { a'Itr' indicates, Ipomoea trifida, 'chr' indicates the chromosome and 'numeric' indicates the position (bp) of each } \\
\text { marker on its respective chromosome. } \\
\text { b'HGs indicates homologous groups. } \\
\text { c's' and 'ds' indicate markers fit to simplex or double simplex segregation in a } \chi^{2} \text {-test, respectively, while 'm' indicates } \\
\text { markers showing significant distortion from simplex or double-simplex segregation. The dosage status of these simplex } \\
\text { and double-simplex markers was further confirmed by NGS read ratio. } \\
\text { d 'Mapped' indicates the 's' and 'ds' SNP markers passed by Lep-MAP3, while, 'Not mapped' indicates the 's' and 'ds' } \\
\text { SNP markers which could not be passed by Filtering2, SeparateChromosomes2, and JoinSingles2All commands. }\end{array}$} \\
\hline
\end{tabular}

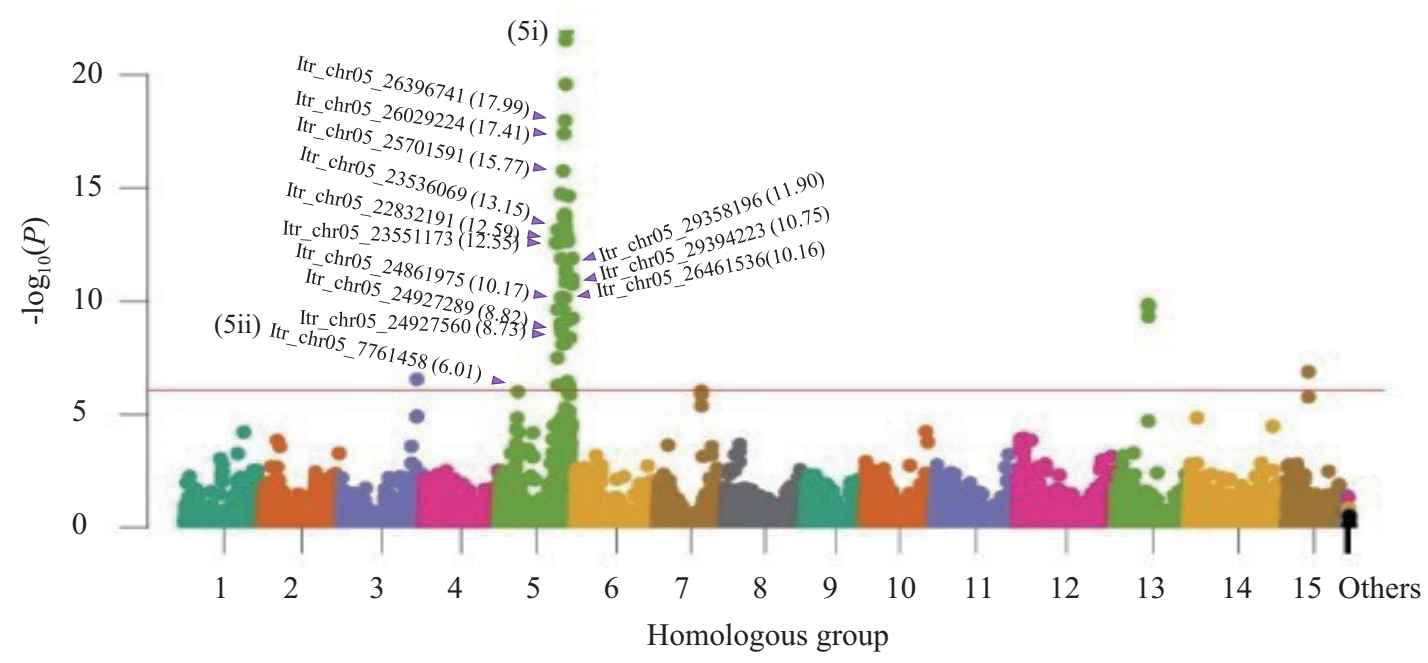

Fig. 3. Manhattan plots from GWAS of the relative AN content. The horizontal redline is the significance threshold of $5 \%$ Bonferroni correction. QTLs related to the relative AN content are indicated. The $-\log _{10}(P)$ for each QTL is shown in parentheses. $5 \mathrm{i}$ and $5 \mathrm{ii}$ indicate the major and smaller signals on HG 5, respectively. 
ratio of white-fleshed clones $(\mathrm{n}=56)$ and the remaining purple-fleshed clones $(\mathrm{n}=38)$ fit to an expected segregation ratio of $1: 1\left(\chi^{2}=3.074, P=\right.$ $0.08)$ or $3: 1\left(\chi^{2}=1.89, P=0.169\right)$ (Fig. 1), which suggests that AN pigmentation of storage roots (including the skin and flesh) in the $\mathrm{KAF}_{1}$ population was determined by a major gene and inherited in a simplex or double simplex manner. However, $\mathrm{A}_{530}$ in purple-fleshed clones showed a continuous distribution (Fig. 2), suggesting that variation in the relative AN content of storage roots in purplefleshed clones was regulated by multiple genes.

\section{GWAS}

GWAS identified six significant signals for the variation of relative $A N$ content in $\mathrm{KAF}_{1}$, with two distinct signals on HG 5 (the major one was indicated as $5 \mathrm{i}$ and the smaller one as 5ii), and one signal each on HGs 3, 7, 13, and 15 (Fig. 3). These six signals consisted of a total of 59 SNPs (Table 1); one in HG $3\left(P=2.78 \times 10^{-07}\right)$, one $(P$ $\left.=9.73 \times 10^{-07}\right)$ and $52\left(P=8.83 \times 10^{-07}-1.11 \times\right.$ $\left.10^{-22}\right)$ in HG 5 , one in HG $7\left(P=9.29 \times 10^{-07}\right)$, three in HG $13\left(P=4.79 \times 10^{-10}-1.38 \times 10^{-10}\right)$, and one in HG $15\left(P=1.30 \times 10^{-07}\right)$.

\section{Map construction and QTL analysis}

A high-density map with 15,747 (26.4\% of the initial 59, 675 SNPs and $55.3 \%$ of the simplex and double-simplex SNPs) markers (a total of 3,411 bins) was constructed on 93 linkage groups (LGs), covering a total distance of $4,726 \mathrm{cM}$. The average length of an LG was $50.8 \mathrm{cM}$ (ranging from 0 to $210.5 \mathrm{cM}$ ), and the average number of SNPs in a single LG was 169.3 (2 to 2759). The marker density of an LG was $0.30 \mathrm{cM}(0.08$ to $2.61 \mathrm{cM})$.

In the QTL analysis of relative AN content, 13 significant SNPs were detected (Table 2). Of these SNPs, 12 were located in the major GWAS signal $5 \mathrm{i}$ on $\mathrm{HG} 5(\mathrm{LOD}=5.27-13.67, \mathrm{PVE}=24.3-62.3 \%)$ and one in the smaller signal 5ii on HG 5 (LOD = 7.52 , PVE $=43.7 \%$ ). However, 10 from the major GWAS signal $5 \mathrm{i}$ and one from the smaller GWAS signal 5 ii were mapped onto a single region of LG 1 $(27.57-58.08 \mathrm{cM})$ as four LOD peaks, whereas two SNPs from the major GWAS signal 5 i were mapped onto another region at $142.10-151.94 \mathrm{cM}$ of LG 12 (Table 2 and Fig. 4). With an increasing effect on

Table 2. SNPs that were confirmed by QTLs analyses for AN in $\mathrm{KAF}_{1}$ progeny

\begin{tabular}{|c|c|c|c|c|c|c|c|c|c|c|}
\hline \multirow[b]{2}{*}{ Marker $^{\mathrm{a}}$} & \multicolumn{3}{|c|}{ Polyploid GWAS } & \multirow[b]{2}{*}{ ANOVA $^{\mathrm{f}}$} & \multicolumn{4}{|c|}{ QTL analysis } & \multicolumn{2}{|c|}{ Genotype score ${ }^{\mathrm{g}}$} \\
\hline & HGs & $\begin{array}{c}-\log _{10} \\
(P)\end{array}$ & $\begin{array}{c}P \text {-value of } \\
\text { GWAS }\end{array}$ & & LOD & LGs & Position & $\begin{array}{l}\text { PVE } \\
(\%)\end{array}$ & KNS & AKM \\
\hline Itr_chr05_24861975 ${ }^{c}$ & $5(5 i)$ & 10.17 & $6.70 \times 10^{-11}$ & $* * *$ & 11.7 & LG 1 & 27.57 & 44.2 & $0.48(\mathrm{G})$ & $4.69(\mathrm{~K})$ \\
\hline Itr_chr05_25701591 ${ }^{b}$ & $5(5 i)$ & 15.77 & $1.69 \times 10^{-16}$ & $* * *$ & 7.68 & LG 1 & 27.57 & 32.7 & $0.27(\mathrm{~T})$ & $5.13(\mathrm{Y})$ \\
\hline Itr_chr05_26461536 ${ }^{d}$ & $5(5 i)$ & 10.16 & $6.96 \times 10^{-11}$ & $* * *$ & 5.27 & LG 1 & 28.11 & 24.3 & $0.78(\mathrm{C})$ & $5.44(\mathrm{Y})$ \\
\hline Itr_chr05_26396741 ${ }^{a}$ & $5(5 i)$ & 17.99 & $1.03 \times 10^{-18}$ & $* * *$ & 9.48 & LG 1 & 40.43 & 37.2 & $0.65(\mathrm{~A})$ & $5.10(\mathrm{R})$ \\
\hline Itr_chr05_24927560 ${ }^{\circ}$ & $5(5 i)$ & 8.73 & $1.85 \times 10^{-09}$ & $* * *$ & 11.77 & LG 1 & 41.51 & 44.7 & $1.24(\mathrm{~T})$ & $5.13(\mathrm{~W})$ \\
\hline Itr_chr05_24927289 ${ }^{c}$ & $5(5 i)$ & 8.82 & $1.52 \times 10^{-09}$ & $* * *$ & 13.47 & LG 1 & 41.51 & 49.4 & $1.24(\mathrm{~A})$ & $5.13(\mathrm{R})$ \\
\hline Itr_chr05_26029224 ${ }^{a}$ & $5(5 i)$ & 17.41 & $3.89 \times 10^{-18}$ & $* * *$ & 12.25 & LG 1 & 44.71 & 46.7 & $1.10(\mathrm{C})$ & $5.19(\mathrm{~S})$ \\
\hline Itr_chr05_23536069 $9^{a}$ & $5(5 i)$ & 13.15 & $7.06 \times 10^{-14}$ & $* * *$ & 10.57 & LG 1 & 50.06 & 40.5 & $0.93(\mathrm{C})$ & $5.39(\mathrm{Y})$ \\
\hline Itr_chr05_23551173 ${ }^{a}$ & $5(5 i)$ & 12.55 & $2.85 \times 10^{-13}$ & $* * *$ & 9.58 & LG 1 & 54.34 & 39.7 & $0.80(\mathrm{C})$ & $4.87(\mathrm{Y})$ \\
\hline Itr_chr05_7761458 ${ }^{a}$ & 5 (5ii) & 6.01 & $9.73 \times 10^{-07}$ & $* * *$ & 7.52 & LG 1 & 54.34 & 43.7 & $1.17(\mathrm{~A})$ & $4.17(\mathrm{~W})$ \\
\hline Itr_chr05_22832191 ${ }^{a}$ & $5(5 \mathrm{i})$ & 12.59 & $2.57 \times 10^{-13}$ & $* * *$ & 9.07 & LG 1 & 58.08 & 35.9 & $0.83(\mathrm{G})$ & $4.72(\mathrm{~K})$ \\
\hline Itr_chr05_29358196 ${ }^{b}$ & $5(5 i)$ & 11.90 & $1.26 \times 10^{-12}$ & $* * *$ & 13.67 & LG 12 & 151.94 & 62.3 & $1.22(\mathrm{C})$ & $5.18(\mathrm{Y})$ \\
\hline Itr_chr05_29394223 ${ }^{a}$ & $5(5 i)$ & 10.75 & $1.77 \times 10^{-11}$ & $* * *$ & 13.67 & LG 12 & 151.94 & 62.3 & $0.97(G)$ & $4.93(\mathrm{R})$ \\
\hline
\end{tabular}

${ }^{a}$ Marker order of the Table 2 was sorted by positions on the physical and genetic maps.

b,c,d,e Indicate the 7, 2, 3, and one SNP loci where QTL analysis and genotype scoring were carried out with 94, 93, 92, and 73 $\mathrm{KAF}_{1}$ genotype data, respectively.

$\mathrm{f}_{* * *}$ indicates significant differences between genotypes by ANOVA at $P<0.001$.

${ }^{\mathrm{g}}$ Arithmetic mean of relative AN content of progenies according to the KNS and AKM genotypes of the SNPs markers, respectively. Alleles determining types of ANs (KNS or AKM) are indicated within the parentheses. 
the relative $\mathrm{AN}$ content, the $11 \mathrm{SNP}$ markers on LG 1 exhibited genotype scores of $0.27-1.24$ and $4.17-5.39$ for the genotypes of KNS and AKM, respectively, while the two SNP markers on LG 12 exhibited the genotype score of $0.97-1.22$ and 4.93 - 5.18 for the genotypes of KNS and AKM, respectively (Table 2). It is noteworthy that out of these 13 SNP loci named in Table 2, there were some missing genotype data for a few SNP loci (Table 2), which may have affected the QTL results.

\section{Locations of SGs and TFs for SNP markers of interest}

By data search, we found that the genes most homologous to $I b F 3 H, I b D F R$, IbMYBI, and IbWD 40 were located on HG 5 of I. trifida pseudomolecule, while the $\mathrm{IbF} 3 \mathrm{H}$ was also located on HG 2 (Table 3). Homologous genes of IbUF3GT and $\mathrm{IbCHI}$ were located on HG 10 and HG 11, respectively. The locations of homologous genes of $I b C H S$ were on HGs 11,12 , and 14, while homologous genes of IbANS and IbbHLH3 were on HG 13 and HG 15, respectively.

\section{Discussion}

Improved breeding tools to facilitate the breeding of sweetpotatoes rich in AN are a priority in Japan, because of the drastic expansion of $\mathrm{AN}$-added products from purple-fleshed sweetpotatoes (Katayama et al. 2017, Tanaka et al. 2017). Here, we have applied the polyploid GWAS method followed by QTL analysis. This enabled us to identify 59 SNP markers linked to the relative AN content on HGs 3, 5 (5i and 5ii), 7, 13, and 15. Specifically, 12 and one
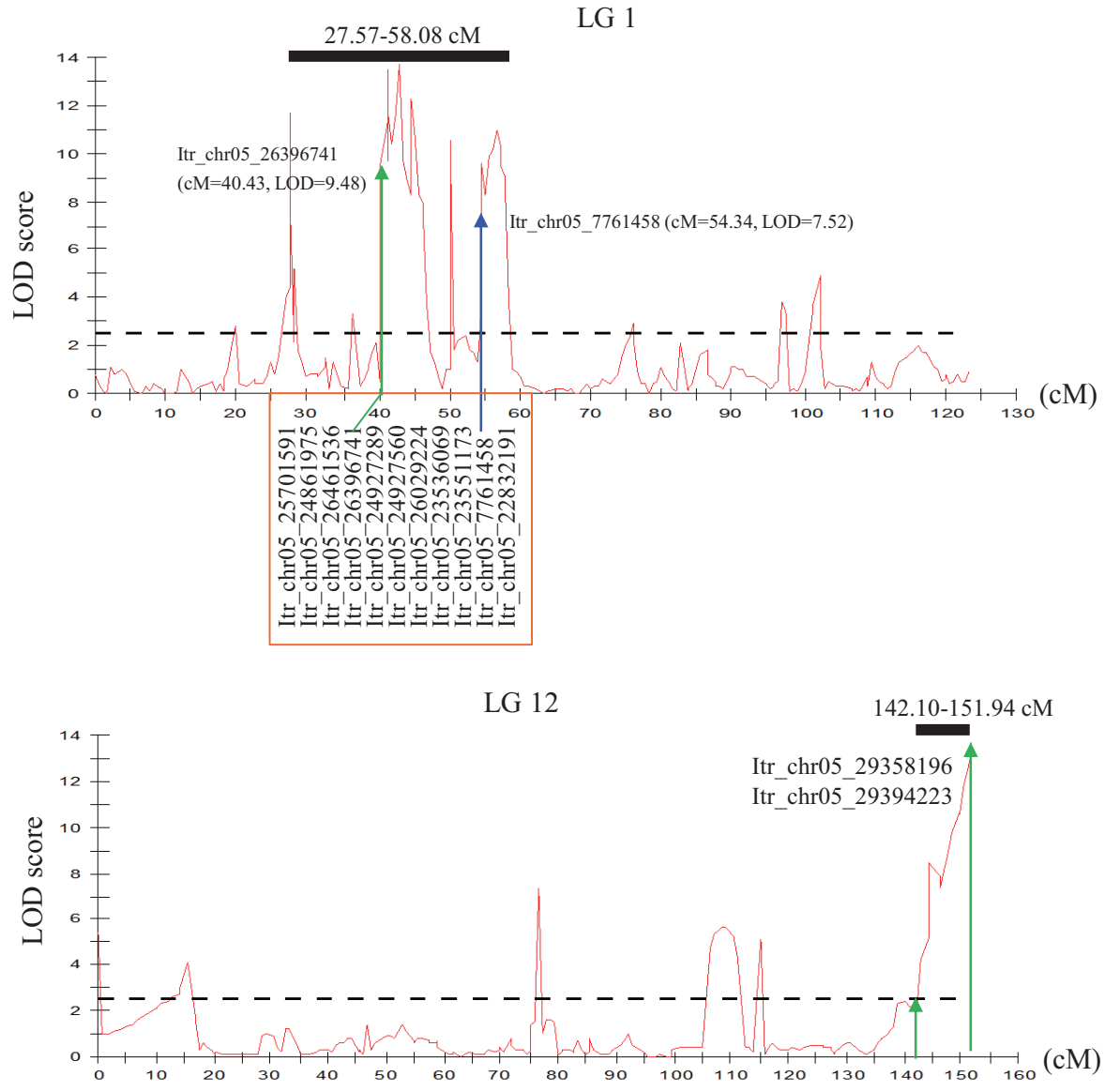

Fig. 4. Detected QTLs with LOD curve on LG 1 and LG 12. The dashed line is the LOD threshold (2.5). On LG 1, the 11 significant SNP loci from the major GWAS signal 5i and the one from the smaller GWAS signal 5ii on HG 5 are shown in the box with the total position covered. The SNP loci which showed the strongest association with relative AN content on the major GWAS signal 5i and the only SNP locus on the smaller GWAS signal 5ii are indicated with green and blue arrowheads, respectively on LG 1. On LG 12, the two associated SNP markers having the same region is indicated with green arrowhead. The respective positions and LOD scores of the indicated QTLs are shown in parentheses. 
SNP markers from the above major signal $5 \mathrm{i}$ and smaller signal 5ii on HG 5, respectively, showed strong associations with the QTL regions, which explained $24.3-62.3 \%$ of the relative $\mathrm{AN}$ variances in this $\mathrm{KAF}_{1}$ progeny (Table 2).

In the GWAS based on the physical map the of $I$. trifida genome, two peaks (5i and 5ii) were detected on HG5. The polyploid GWAS method (Yamamoto et al. 2020) has a merit that error-tolerance genotyping scores, allele dosage probability, calculated from low coverage data are employed for genetic analysis. On the other hand, in genetic mapping, the SNPs at the 5i and 5ii were mapped together in the single region at $27.57-58.08 \mathrm{cM}$ of LG 1 as four LOD peaks. This conflict could be arisen by possible chromosome rearrangements between I. trifida and I. batatas. Another possibility might be genotyping errors due to the low-coverage data. Genotyping scores were required in the QTL mapping with MapQTL even if the read coverages were insufficient to distinguish three scores, e.g., A, $\mathrm{B}$, and $\mathrm{H}$. To obtain high accuracy scores in polyploid species, cost-consuming high-coverage data would be required. Obviously, this situation is not desired in practical breeding programs. One of the reasons that fewer SNPs were associated with QTL regions might be that the QTL analysis method used in this study was limited to the mode of inheritance of Mendelian markers that resembled the mode of inheritance for the simplex and double simplex dosages of markers. In our analysis, approximately $52.3 \%$ of SNP markers were discarded by the $\chi^{2}$-test, i.e., among the 59,675 SNP markers, 31,183 were not likely simplex and double- simplex markers because the observed segregation pattern was significantly distorted from the expected segregation ratio for simplex or double-simplex markers. Furthermore, only 55.3\% (15,747 out of 28,492 SNPs) of the simplex and double-simplex dosages of markers could be mapped by Lep-MAP3. There might be a handful of reasons of why the remaining $44.7 \%$ of markers were not mapped, including the autohexaploid genome nature of the sweetpotato, which could easily lead to complex recombination among homologous chromosomes in the male and female gametes. As categorized in Table 1, out of 59 SNPs, 28 were simplex and double-simplex. However, only 13 markers were mapped by Lep-MAP3, and unfortunately the remaining 15 markers were discarded by LepMAP3. It is likely that polyploid GWAS minimized the loss of SNP information during the genetic analysis compared to the current QTL analysis. However, GWAS does have the potential for falsepositive error, and validation of the results is necessary (Zhu et al. 2008, Korte and Farlow 2013). The number of markers used in the GWAS highly affects its results (Han et al. 2018), particularly in the case of SNP markers where numerous markers are produced by NGS-based genotyping in a single experiment. Thus, the increase in the number of SNPs could easily lead to a genome-wide higher number of false-positive signals. Associations at unlinked, noncausal markers can also arise because of the inheritance pattern of the given trait (qualitative or quantitative), pleiotropy, epistasis, etc. (Platt et al. 2010). Although polyploid GWAS identified a much higher number of SNPs linked to

Table 3. Locations of structural genes (SGs) and transcription factors (TFs) associated with AN biosynthesis

\begin{tabular}{ccc}
\hline Name & Homologous groups & Position within I. trifida \\
\hline$I b M Y B 1$ & 5 & $2573593-2574056$ \\
\hline$I b W D 40$ & 5 & $29809214-29807853$ \\
\cline { 2 - 3 }$I b F 3 H$ & 5 & $1716060-1716489$ \\
\hline$I b D F R$ & 2 & $4297086-4297516$ \\
\hline$I b U F 3 G T$ & 5 & $1988121-1988466$ \\
\hline$I b C H I$ & 10 & $1245564-1244686$ \\
\hline$I b C H S$ & 11 & $29751152-29751481$ \\
\hline$I b A N S$ & 11 & $5153677-5154640$ \\
\hline$I b b H L H 3$ & 12 & $6374874-6375977$ \\
\hline & 14 & $31216008-31216989$ \\
\hline
\end{tabular}


the relative AN content compared to QTL analysis, it should be born in mind that the smaller GWAS signals, other than the smaller signal 5ii on HG 5, need to be confirmed in a future study.

In total, further genetic analysis using highcoverage NGS data and a mapping algorithm optimized to heterozygous autopolyploid would be necessary to determine the exact number and location of the genetic regions associated with AN.

To gain insight into the candidate gene from $\mathrm{HG}$ 5 that is homologous to the AN biosynthesis gene, we have interpreted our database search results in which TFs $I b M Y B I$ and $I b W D 40$ and SGs $I b F 3 H$ and $I b D F R$ were found to be located on HG 5 (Table $3)$. This result suggests that an SNP marker or markers out of those 13 SNPs from HG 5 might include candidate gene(s) homologous to one or all of these TFs and SGs. Among these TFs and SGs, MYB TF has been well characterized in other plant species as well as in the sweetpotato. A retrotransposon-induced mutation on the promoter of the MYB gene created white-skinned grape (Vitis vinifera) cultivars from red-skinned ones (Kobayashi et al. 2004). Recently, heterologous expression of the IbMYBI gene in the orange-fleshed sweetpotato increased AN pigment accumulation in the storage root (Park et al. 2015). Moreover, Mano et al. (2007) reported that IbMYBI was predominant in the purple-fleshed tuberous roots of the Ayamurasaki cultivar rich in a higher level of AN. The authors also reported that the expression of sweetpotato IbMYBI alone was sufficient for the induction of all SGs involved in AN biosynthesis and $\mathrm{AN}$ accumulation in the flesh of tuberous roots, as well as in heterologous tissues or heterologous plant species. Later, Tanaka et al. (2012) reported that there are three variants of the $I b M Y B 1$ gene in the purple-fleshed Ayamurasaki, namely IbMYB1-1, $I b M Y B 1-2 a$, and IbMYB I-2b, where IbMYB I-2a and $I b M Y B 1-2 b$, but not IbMYB1-1, were tightly linked to $\mathrm{AN}$ accumulation in the storage roots. Thus, IbMYB1 may be involved in AN pigmentation accumulation in purple-fleshed $\mathrm{KAF}_{1}$ through the regulation of SGs (Fig. 1 and Fig. 2). WD40, which is a member of the TF complex MYB-bHLH-WD40 (MBW), has been shown to functionally enhance complex activation rather than to directly participate in the recognition of the target gene promoter (Baudry et al. 2004). Although in transgenic Arabidopsis thaliana seedlings, IbWD40 from the purple-fleshed sweetpotato was suggested to regulate AN biosynthesis (Dong et al. 2014), there is no evidence so far based on a mutant or overexpressed line in the sweetpotato that IbWD40 regulates AN biosynthesis. In light of the above discussion, we postulated that IbMYB1 might be harbored by the HG 5 as a major factor in determining $\mathrm{AN}$ accumulation in the storage roots via the regulation of these co-located or other AN biosynthesis genes. Further study is necessary to clarify the above hypothesis.

\section{Conclusion}

Here, we located 13 SNP markers on HG 5. One of them was assumed to include the candidate gene homologous to any of the TFs and AN SGs that acts as a major factor in determining $\mathrm{AN}$ accumulation in the storage root of sweetpotato. These results enhance our understanding of the underlying genetic basis of AN accumulation in the storage roots of sweetpotatoes, and the SNP markers generated in this study could be a platform for future markerassisted selection for this trait in a sweetpotato breeding program. However, further analysis is necessary to confirm and develop molecular markers for these SNPs.

\section{Acknowledgments}

The authors wish to thank M. Nishinaka (Institute of Crop Science, NARO, Ibaraki, Japan), Y. Okada (KARC/NARO, Okinawa, Japan), A. Oyanagi (KARC/NARO, Miyazaki, Japan) and Y. Monden (Graduate School of Environmental and Life Science, Okayama University, Japan) for their suggestions. We also thank A. Tokiwa, A. Tsuneyoshi, M. Kamimura (sweetpotato crossing and seed production team) and the members of the field management staff of Miyakonojo research station, KARC/NARO for their assistance.

\section{References}

Baudry A, Heim MA, Dubreucq B, Caboche M, Weisshaar B, Lepiniec L 2004 TT2, TT8, and TTG1 synergistically specify the expression of BANYULS and proanthocyanidin biosynthesis in Arabidopsis thaliana. Plant J. 39: 366380.

Cervantes-Flores JC, Sosinski B, Pecota KV, Mwanga ROM, Catignani GL, Truong VD, Watkins RH, Ulmer MR, Yencho GC 2011 Identification of quantitative trait loci for dry-matter, starch, and $\beta$-carotene content in sweetpotato. Mol. Breed. 28: 201-216.

Dong W, Niu L, Gu J, Gao F 2014 Isolation of a WD40repeat gene regulating anthocyanin biosynthesis in storage roots of purple-fleshed sweet potato. Acta Physiol. Plant. 36: 1123-1132.

Food and Agricultural Organization (FAO) 2018 FAO Statistics. http://www.fao.org/faostat/en/\#data/QC (latest update: September 14, 2020), (accessed September 29, 2020)

Gemenet DC, Pereira GDS, De Boeck B, Wood JC, 
Mollinari M, Olukolu BA, Diaz F, Mosquera V, Ssali RT, David M, Kitavi MN, Burgos G, Felde TZ, Ghislain M, Carey E, Swanckaert J, Coin LJM, Fei Z, Hamilton JP, Yada B, Yencho GC, Zeng Z-B, Mwanga ROM, Khan A, Gruneberg WJ, Buell CR 2020 Quantitative trait loci and differential gene expression analyses reveal the basis for negatively-associated $\beta$-carotene and starch content in hexaploid sweetpotato [Ipomoea batatas (L.) Lam.]. Theor. Appl. Genet. 133: 23-36.

Han K, Lee H-Y, Ro N-Y, Hur O-S, Lee J-H, Kwon J-K, Kang B-C 2018 QTL mapping and GWAS reveal candidate genes controlling capsaicinoid content in Capsicum. Plant Biotechnol. J. 16: 1546-1558.

Haque E, Tabuchi H, Monden Y, Suematsu K, Shirasawa K, Isobe S, Tanaka M 2020 QTL analysis and GWAS of agronomic traits in sweetpotato (Ipomoea batatas L.) using genome wide SNPs. Breed. Sci. 70: 283-291.

Hirakawa H, Okada Y, Tabuchi H, Shirasawa K, Watanabe A, Tsuruoka H, Minami C, Nakayama S, Sasamoto S, Kohara M, Kishida Y, Fujishiro T, Kato M, Nanri K, Komaki A, Yoshinaga M, Takahata Y, Tanaka M, Tabata S, Isobe S 2015 Survey of genome sequences in a wild sweet potato, Ipomoea trifida (H.B.K.) G. Don. DNA Res. 22: 171-179.

Katayama K, Kobayashi A, Sakai T, Kuranouchi T, Kai Y 2017 Recent progress in sweetpotato breeding and cultivars for diverse applications in Japan. Breed. Sci. 67: 3-14.

Kobayashi S, Goto-Yamamoto N, Hirochika H 2004 Retrotransposon-induced mutations in grape skin color. Science 304: 982.

Korte A, Farlow A 2013 The advantages and limitations of trait analysis with GWAS: a review. Plant Methods 9: 29.

Kumagai T 2001 Breeding and varieties of sweetpotato. Farming Japan 35: 11-17.

Mano H, Ogasawara F, Sato K, Higo H, Minobe Y 2007 Isolation of a regulatory gene of anthocyanin biosynthesis in tuberous roots of purple-fleshed sweet potato. Plant Physiol. 143: 1252-1268.

Nakayama H, Tanaka M, Takahata Y, Matsui K, Iwahori H, Sano Z, Yoshinaga M 2012 Development of AFLPderived SCAR markers associated with resistance to two races of southern root-knot nematode in sweetpotato. Euphytica 188: 175-185.

Park S-C, Kim Y-H, Kim SH, Jeong YJ, Kim CY, Lee JS, Bae J-Y, Ahn M-J, Jeong JC, Lee H-S, Kwak S-S 2015 Overexpression of the IbMYBI gene in an orangefleshed sweet potato cultivar produces a dual-pigmented transgenic sweet potato with improved antioxidant activity. Physiol. Plant. 153: 525-537.

Platt A, Vilhjálmsson BJ, Nordborg M 2010 Conditions under which genome-wide association studies will be positively misleading. Genetics 186: 1045-1052.

Rastas P 2017 Lep-MAP 3: robust linkage mapping even for low-coverage whole genome sequencing data. Bioinformatics 33: 3726-3732.

Sakai T, Kumagai T, Kai Y, Ishiguro K, Yamakawa O, Katayama K, Nakazawa Y, Yoshinaga M 2010
'Akemurasaki': A new sweetpotato cultivar. Bull. Natl. Agric. Res. Cent. Kyushu Okinawa Reg. 53: 1-24.

Sasai R, Tabuchi H, Shirasawa K, Kishimoto K, Sato S, Okada Y, Kuramoto A, Kobayashi A, Isobe S, Tahara M, Monden Y 2019 Development of molecular markers associated with resistance to Meloidogyne incognita by performing quantitative trait locus analysis and genomewide association study in sweetpotato. DNA Res. 26: 399-409.

Shirasawa K, Hirakawa H, Isobe S 2016 Analytical workflow of double-digest restriction site-associated DNA sequencing based on empirical and in silico optimization in tomato. DNA Res. 23: 145-153.

Shirasawa K, Tanaka M, Takahata Y, Ma D, Cao Q, Liu Q, Zhai H, Kwak S-S, Cheol Jeong JC, Yoon U-H, Lee H-U, Hirakawa H, Isobe S 2017 A high-density SNP genetic map consisting of a complete set of homologous groups in autohexaploid sweetpotato (Ipomoea batatas). Sci. Rep. 7: 44207.

Tanaka M, Ishiguro K, Oki T, Okuno S 2017 Functional components in sweetpotato and their genetic improvement. Breed. Sci. 67: 52-61.

Tanaka M, Takahata Y, Kurata R, Nakayama H, Yoshinaga M 2012 Structural and functional characterization of IbMYB 1 genes in recent Japanese purple-fleshed sweetpotato cultivars. Mol. Breed. 29: 565-574.

TRAS sweetpotato genome sequencing consortium 2019 Sweetpotato genome sequencing efforts by the TRAS community. Proceeding of Plant Animal Genome XXVII, W1034.

Turner SD 2014 qqman: an R package for visualizing GWAS results using QQ and manhattan plots. bioRxiv 005165

Yada B, Brown-Guedira G, Alajo A, Ssemakula GN, Owusu-Mensah E, Carey EE, Mwanga ROM, Yencho GC 2017 Genetic analysis and association of simple sequence repeat markers with storage root yield, dry matter, starch and $\beta$-carotene content in sweetpotato. Breed. Sci. 67: 140-150.

Yamamoto E, Shirasawa K, Kimura T, Monden Y, Tanaka M, Isobe S 2020 Genetic mapping in autohexaploid sweet potato with low-coverage NGS-based genotyping data. G3. 10: 2661-2670.

Yu X, Zhao N, Li H, Jie Q, Zhai H, He S, Li Q, Liu Q 2014 Identification of QTLs for starch content in sweetpotato (Ipomoea batatas (L.) Lam.). J. Integr. Agric. 13: 310315.

Zhang K, Wu Z, Tang D, Lv C, Luo K, Zhao Y, Liu X, Huang Y, Wang J 2016 Development and identification of SSR markers associated with starch properties and $\beta$-carotene content in the storage root of sweet potato (Ipomoea batatas L.). Front. Plant Sci. 7: 223.

Zhao N, Yu X, Jie Q, Li H, Li H, Hu J, Zhai H, He Q, Liu Q 2013 A genetic linkage map based on AFLP and SSR markers and mapping of QTL for dry-matter content in sweetpotato. Mol. Breed. 32: 807-820.

Zhu C, Gore M, Buckler ES, Yu J. 2008 Status and prospects of association mapping in plants. Plant Genome 1: 5-20. 